\title{
Introduction
}

Pregnancy is characterized by increased intestina calcium absorption, normal ionized or albumincorrected calcium, high calcitriol, low parathyroid hormone (PTH), gradually increasing PTH related peptide (PTHrP), and hypercalciuria (Fig. 1).

These differing hormonal changes can lead to nonclassic presentations of disorders of bone and mineral metabolism.

\section{Hypercalcaemia in Pregnancy}

\section{Normal pregnancy is} associated with many nonspecific symptoms that overlap with symptoms of

hypercalcaemia, such as nausea,

hyperemesis,

constipation, fatigue, weakness, and mental symptoms.

- The overlap of symptoms may delay the diagnosis of hypercalcaemia and/or hyperparathyroidism.

Stillbirth, miscarriage, and neonatal tetany remain among the serious and common complications of hypercalcaemia. Primary

hyperparathyroidism (PHPT) is the most common cause of hypercalcaemia in pregnancy.

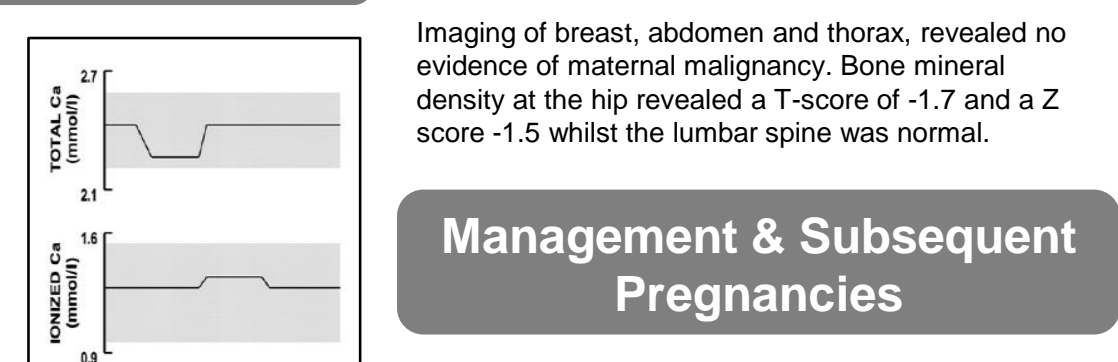

Intravenous fluid therapy led to short lived symptomatic improvement. Short course of prednisolone failed to suppress serum calcium.

Safety of bisphosphonates during pregnancy is not established. Decision was made for medical termination of pregnancy (TOP) at 17 weeks from lack of symptomatic control of hypercalcaemia and the concern of its effect on the foetus.

Calcium, PTHrP and 1,25OH-VitD levels normalised post termination. Foetal autopsy revealed no abnormalities apart from placental membrane calcification. The risks of gestational hypercalcaemia in future pregnancies discussed and advised earlier monitoring of calcium.

She conceived again and developed hypercalcaemia elevated $1,25 \mathrm{OH}$-VitD and variably elevated PTHrP. Hypercalcaemia was uncontrolled, requiring termination (12 weeks) with subsequent normalisation of calcium metabolic parameters. Placental analysis failed to show abnormal PTHrP staining.

\section{Extensive counselling undertaken regarding future} pregnancies. There was no hypercalcaemia in previous pregnancies with former partner.

The patient and her current partner keen to pursue further pregnancy. In 2008, she conceived again with a miscarriage at 8 weeks. She had another TOP in 2013 due to gestational hypercalcaemia.

The usual causes of hypercalcaemia were excluded. This appears to be a pregnancy-related phenomenon, and postulated mechanisms are either excessive calcium gut absorption due to increased sensitivity to 1,25 Vitamin D or an occult source.

There are currently limited studies assessing the role of bisphosphonates in pregnancy or other potential calcium lowering therapies (e.g. denosumab). possibility of increased placental 1- $\alpha$ hydroxylase enzyme activation.

Aberrant prolactin receptors and its responsiveness to physiological levels of prolactin may increase PTH with altered gene transcriprition.

The role of bisphosphonate or denosumab for hypercalcaemia in such circumstances needs further research. Her calcium homeostasis returns to normality immediately post termination.

\section{References}

- Kovacs CS. Calcium and bone metabolism disorders during pregnancy and lactation. Endocrinol Metab Clin N Am 40 (2011) 795-826.

- Mastaglia SR, et al. Intravenous bisphosphonate treatment and pregnancy: its effects on mother and bone health. Osteoporosis Int (2010).

- Levy S, et al. Pregnancy outcome following utero exposure to bisphosphonate. Bone (2009).

- Ornoy A, et al. The outcome of pregnancy following prepregnancy or early pregnancy alendronate treatment. Reprod Toxicol (2006) 578-579. is discharged and as the haemodilution of pregnancy resolves.

- Neonatal hypocalcemia and other complications are not inevitable after marked maternal hypercalcemia during pregnancy. 